\title{
Phenotypic Characterization of Multidrug-resistant Escherichia Coli with Special Reference to Extended-spectrum-beta-lactamases and Metallo-beta-lactamases in a Tertiary Care Center
}

Basudha Shrestha, ' Shovita Shrestha, ' Shyam Kumar Mishra, ${ }^{1}$ Hari Prasad Kattel,' Tatsuya Tada, ${ }^{2}$ Hiroshi Ohara Teruo Kirikae, ${ }^{2}$ Basista Prasad Rijal,' Jeevan Bahadur Sherchand, ' Bharat Mani Pokhrel'

'Department of Microbiology, Institute of Medicine, Maharajgunj, Kathmandu, Nepal, ${ }^{2}$ Department of Infectious Diseases, Research Institute and ${ }^{3}$ Department of International Medical Cooperation, National Center for Global Health and Medicine, Tokyo, Japan

\section{ABSTRACT}

Introduction: The increasing reports on extended-spectrum-beta-lactamase and metallo-betalactamase producing Escherichia coli have addressed a potential threat to global health since it is found to be highly resistance to most of the currently available antibiotics including carbapenems. The present study was aimed to determine the antibiogram of extended-spectrum-beta-lactamase and metallo-beta-lactamase producing MDR E. coli isolates from various clinical samples.

Methods: This was a cross-sectional study conducted over a period of seven months (December 2013 to July 2014) at bacteriology laboratory of Tribhuvan University Teaching Hospital. A total of 250 clinical specimens (urine, pus, sputum, blood, body fluid, bile, tissue and central venous pressure line tip) were processed from inpatients, with multidrug-resistant (MDR) Escherichia coli infections. Standard microbiological techniques were used for isolation and identification of the isolates. The presence of extended-spectrum-beta-lactamase was detected by phenotypic confirmatory test recommended by Clinical and Laboratory Standards Institute and imipenem (IMP) / EDTA combined disc method was performed to detect metallo-beta-lactamase mediated resistance mechanism.

Results: We found high level of beta lactamase mediated resistance mechanism as part of multidrug resistance. Among $250 \mathrm{MDR}$ isolates, $60 \%$ isolates were extended-spectrum-beta-lactamase producers and $17.2 \%$ isolates were metallo-beta-lactamase producers. Co-existence of extended-spectrum-betalactamase and metallo-beta-lactamase identified in $6.8 \%$ isolates.

Conclusions: Beta-lactamase mediated resistance mechanisms are accounting very high in the multidrug resistant isolates of $E$. coli. Therefore, early detection of beta lactamase mediated resistant strains and their current antibiotic susceptibility pattern is necessary to avoid treatment failure and prevent the spread of MDR.

Keywords: e. coli; extended-spectrum- $\beta$-lactamase; metallo- $\beta$-lactamase; multidrug-resistance.

\section{INTRODUCTION}

The production of beta-lactamases ( $\beta$-lactamases) is the most common mechanism responsible for resistance to $\beta$-lactams among clinical isolates of Enterobacteriaceae family. ${ }^{1}$ The $\beta$-lactamases receiving the most attention are the extended-spectrum-beta-lactamases (ESBLs), plasmid-mediated AmpC $\beta$-lactamases and carbapenemases because of rapid global dissemination of these enzymes. ${ }^{1,2}$ ESBLs confer bacterial resistance to all $\beta$-lactams except carbapenems and cephamycins, which are inhibited by $\beta$-lactamase inhibitors such as clavulanic acid. ${ }^{3}$ Carbapenemases consist of serine$\beta$-lactamases (KPC, OXA, GES, etc.) and Metallo- $\beta$ lactamases (MBLs) which are associated with resistance to aminoglycosides and fluoroquinolones. ${ }^{4}$

Correspondence: Basudha Shrestha, Microbiology Department, Maharaiguni Medical Campus, Institute of Medicine, Maharajguni, Kathmandu, Nepal. Email: basudha111@hotmail.com, Phone: +977. 9851030594 
Shrestha et al. Phenotypic Characterization of Multidrug-Resistant Escherichia Coli with Special Reference...

E.coli has been reported as opportunistic, worrisome, nosocomial and community-associated pathogen and the most frequent isolate in various clinical specimens. ${ }^{5}$ E.coli is a major concern in medical community because of worldwide emergence of MDR strains mediated by ESBL and MBL enzymes. . $^{3,6,7}$

Little information is currently available regarding ESBL and MBL producing E.coli in Nepal. Keeping in view the above background, this cross-sectional study was conducted to provide information on antibiotic susceptibility with special reference to ESBL and MBL in MDR E.coli isolates from hospitalized patients.

\section{METHODS}

A cross-sectional study was conducted at the bacteriology laboratory of Tribhuvan University Teaching Hospital (TUTH), Kathmandu, over a period of seven months from $12^{\text {th }}$ December 2013 to $12^{\text {th }}$ July 2014. This study was approved by Institutional Review Board of Institute of Medicine. A total of 250 consecutive non-repetitive multidrug-resistant Escherichia coli (MDR E. coli) were isolated from various clinical samples such as urine $(n=127)$, pus $(n=65)$, sputum $(n=37)$, blood $(n=9)$, body fluid $(n=4)$, bile $(n=5)$, tissue $(n=2)$ central venous pressure (CVP) line $(n=1)$ which were received from hospitalized patients. The isolation and identification of Escherichia coli were performed following standard microbiological techniques as described by American Society of Microbiology (ASM). ${ }^{8}$ Data were analyzed using Microsoft Excel 2007.

\section{Antimicrobial susceptibility testing}

Antibiotic susceptibilities were determined by KirbyBauer disk diffusion method and the results were interpreted according to the guidelines of the Clinical Laboratory Standard Institute (CLSI). ${ }^{9}$ The antibiotic discs used were amikacin (30 $\mu \mathrm{g})$, amoxycillin (10 $\mu \mathrm{g})$, amoxycillin/clavulanic acid $(20 / 10 \mu \mathrm{g})$, cefepime (30 $\mu \mathrm{g})$, cefoperazone/sulbactam $(75 / 30 \mu \mathrm{g})$ ceftazidime (30 $\mu \mathrm{g})$, cefoxitin $(30 \mu \mathrm{g})$, ceftriaxone (30 $\mu \mathrm{g})$, chloramphenicol (30 $\mu \mathrm{g})$, ciprofloxacin (5 $\mu \mathrm{g})$, colistin sulphate $(10 \mu \mathrm{g})$, cotrimoxazole $(1.25 / 23.75 \mu \mathrm{g})$, doxycycline $(30 \mu \mathrm{g})$, gentamicin (10 $\mu \mathrm{g})$, imipenem(10 $\mu \mathrm{g})$, meropenem $(10 \mu \mathrm{g})$, nitrofurantoin (300 $\mu \mathrm{g})$, ofloxacin (5 $\mu \mathrm{g})$, piperacillin/tazobactam (100/10 $\mu \mathrm{g})$, polymyxin-B (300 units) and tigecycline (5 $\mu \mathrm{g})$.

\section{Definition of MDR}

MDR E.coli were defined as the isolates of E.coli resistant to at least three classes of antimicrobial agents-all penicillins and cephalosporins (including inhibitor combinations), aminoglycosides, cephamycins, fluoroquinolones, folate pathway inhibitors, glycylcyclines, phenicol, polymyxins and tetracyclines. ${ }^{10}$

\section{Tests for ESBL-production}

All of the 250 isolates were screened for ESBL production by CLSI phenotypic confirmatory test of combined disc assay method. ${ }^{9}$ One disc of ceftazidime $(30 \mu \mathrm{g})$ alone and one in combination with clavulanic acid (30 $\mu \mathrm{g} / 10 \mu \mathrm{g}$ ) were placed at a distance of $20 \mathrm{~mm}$ on a Muller Hinton agar plate inoculated with a bacterial suspension of $0.5 \mathrm{McF}$ arland turbidity standards, and incubated overnight at $37^{\circ} \mathrm{C}$. The ESBL-producing strains showed a variation greater than $5 \mathrm{~mm}$ between the inhibition zones around cefotaxime or ceftazidime discs alone in comparison with the inhibition zone around cefotaxime/clavulanic acid or ceftazidime/ clavulanic acid discs. Klebsiella pneumoniae ATCC 700603 and E. coli ATCC 25922 were used as positive and negative control strains respectively.

\section{Tests for MBL-production}

Screening test: The isolates were subjected for MBL detection when the zone of inhibition (ZOI) for ceftazidime (CAZ) $(30 \mu \mathrm{g})$ was $<18 \mathrm{~mm}$. The sensitivity or resistivity pattern to imipenem (IPM) $(10 \mathrm{mg})$ and or meropenem (MEM) $(10 \mu \mathrm{g})$ were not considered for MBL detection as bacteria might harbour "hidden MBL" and if only the carbapenem resistant phenotypes were considered, then such hidden $\mathrm{MBL}$ carrying isolates would be missed.

MBL confirmation by combination disk method: All 250 isolates were phenotypically confirmed for metallo$\beta$-lactamase production as described by Franklin et al. ${ }^{11}$ Briefly, two imipenem (IPM) disks $(10 \mu \mathrm{g})$, one containing $10 \mu \mathrm{l}$ of $0.1 \mathrm{M}(292 \mu \mathrm{g})$ anhydrous EDTA (Sigma Chemicals, St. Louis, MO), were placed $25 \mathrm{~mm}$ apart (center to center). An increase in zone diameter of $>4 \mathrm{~mm}$ around the IPM/EDTA disk compared to that of the IPM disk alone was considered positive for an MBL. For MBL test standardization, Pseudomonas aeruginosa ATCC 27853 was used as a negative control strain and Pseudomonas aeruginosa PA 105663 was used as a positive control.

\section{RESULTS}

Two hundred and fifty specimens with MDR E. coli infection were included in this study. These included ( $n=127,50.8 \%)$ urine, $(n=65,26 \%)$ pus, $(n=36$, $14.4 \%)$ sputum, $(n=9,3.6 \%)$ blood, $5(2.0 \%)$ bile, ( $n=4,1.6 \%)$ body fluid, $(n=2,0.8 \%)$ tissue, $(n=1$, $0.4 \%)$ endotracheal secretion and $(n=1,0.4 \%)$ CVP line.

The highest number of MDR E. coli $(\mathrm{n}=141,56.4 \%$ ) was isolated from surgical wards (Table 1). 
Shrestha et al. Phenotypic Characterization of Multidrug-Resistant Escherichia Coli with Special Reference...

\begin{tabular}{|ll|}
\hline $\begin{array}{l}\text { Table 1. Prevalence of MDR } \\
\text { (n= } \text {. } \text { coli in } \text { in } \text { different wards }\end{array}$ \\
\hline Wards & No $(\%)$ \\
Surgical & $141(56.4)$ \\
ICU & $21(8.4)$ \\
Medical & $18(7.2)$ \\
Neuro & $18(7.2)$ \\
Pediatric & $14(5.6)$ \\
Post-operative & $14(5.6)$ \\
Maternity & $7(2.8)$ \\
Nephro & $7(2.8)$ \\
Orthopedics & $5(2.0)$ \\
Pediatric ICU & $2(0.8)$ \\
Burn & $1(0.4)$ \\
Eye & $1(0.4)$ \\
Surgical ICU & $1(0.4)$ \\
\hline
\end{tabular}

\section{Antibiogram of MDR E.coli}

Out of 21 antibiotics used for antibiotic susceptibility test, colistin sulphate, polymyxin- $B$ and tigecycline were $100 \%$ effective followed by imipenem $(n=204$, $81.6 \%)$, chloramphenicol $(n=90,73.6 \%)$, amikacin $(n=170,68 \%)$, meropenem ( $n=149,59.6 \%)$ and piperacillin-tazobactam $(n=146,58.4 \%)$. All isolates were $100 \%$ resistance to amoxycillin, cefotaxime, ceftazidime, and ceftriaxone (Table 2).

\section{Distribution of ESBL and MBL in MDR E .coli}

Out of 250 E. coli strains studied, $(n=239,95.6 \%)$ strains produced any of the 2 types of $\beta$ - lactamases i.e. $\mathrm{ESBL}$ and $\mathrm{MBL}$, either alone or co-producer (Figure $2)$. In our study, $(\mathrm{n}=15,6.2 \%)$ E. coli strains were positive for both types of $\beta$-lactamases i.e. ESBL and $\mathrm{MBL}$ in combination.

Pattern of ESBL and MBL producers E. coli in different clinical specimens

Out of total $150 \mathrm{ESBL}$ positive $E$. coli strains, maximum ( $n=91,60.6 \%$ ) strains were isolated from urine followed by $(n=31,20.6 \%)$ strains from pus, and $(n=18,12.0 \%)$ from sputum. Out of $43 \mathrm{MBL}$ producers, $(n=17,39.5 \%)$ were isolated from urine and $(n=14,32.5 \%)$ were isolated from pus. No MBL producing $E$. coli strain was isolated from body fluids and tissue (Table 3).

Distribution of ESBL and MBL- producers in different wards

ESBL and MBL producing MDR E. coli were the most common in surgical wards. Out of 250 MDR E. coli isolates, $(n=80,53.3 \%)$ ESBL-producers and $(n=26$, $60.4 \%) \mathrm{MBL}-$ producers were isolated from surgical wards (Table 4).

\begin{tabular}{|lcc|}
\hline \multicolumn{3}{|c|}{ Table 2. Antibiogram of MDR E.coli $(\mathbf{n}=\mathbf{2 5 0 )}}$. \\
\hline Antibiotics & Sensitive & Resistant \\
Colistin sulphate & $250(100.0)$ & No (\%) \\
Polymyxin-B & $250(100.0)$ & $0(0.0)$ \\
\hline Tigecycline & $250(100.0)$ & $0(0.0)$ \\
Imipenem & $204(81.6)$ & $46(18.4)$ \\
Chloramphenicol * & $90(73.8)$ & $33(26.2)$ \\
Amikacin & $170(68.0)$ & $80(32.0)$ \\
Meropenem & $149(59.6)$ & $101(40.4)$ \\
Nitrofurantoin* & $75(59.0)$ & $28(41.0)$ \\
Piperacillin /tazobactam & $146(58.4)$ & $104(41.6)$ \\
Gentamicin & $108(43.2)$ & $142(56.8)$ \\
Cefoperazone / & $102(40.8)$ & $148(59.2)$ \\
sulbactam & & \\
Doxycycline & $63(25.2)$ & $187(74.8)$ \\
Cefoxitin & $74(29.6)$ & $176(70.4)$ \\
Amoxycillin/ clavulanic & $64(25.6)$ & $186(74.4)$ \\
acid & & \\
Cefepime & $23(9.2)$ & $227(90.8)$ \\
Cotrimoxazole & $23(9.2)$ & $227(90.8)$ \\
Ciprofloxacin & $10(4.0)$ & $240(96.0)$ \\
Ofloxacin & $10(4.0)$ & $240(96.0)$ \\
Ceftazidime & $1(0.4)$ & $249(99.6)$ \\
Cefotaxime & $0(0.0)$ & $250(100.0)$ \\
Ceftriaxone & $0(0.0)$ & $250(100.0)$ \\
Amoxycillin & $0(0.0)$ & $250(100.0)$ \\
\hline$*$ (10)
\end{tabular}

** Except for urinary isolates

* Only for urinary isolates

Table 3. Isolation of ESBL and MBL producers E.coli from different clinical specimens.

\begin{tabular}{|llll|}
\hline \multirow{2}{*}{ Specimen } & ESBL & MBL & ESBL + MBL \\
& No (\%) & No. (\%) & No. (\%) \\
Urine & $91(60.6)$ & $18(41.8)$ & $9(60.0)$ \\
Pus & $31(20.7)$ & $14(32.5)$ & $2(13.3)$ \\
Sputum & $18(12.0)$ & $8(18.6)$ & $4(26.7)$ \\
Blood & $4(2.7)$ & $2(4.6)$ & $0(0.0)$ \\
Body fluid & $3(2.0)$ & $0(0.0)$ & $0(0.0)$ \\
Tissue & $2(1.3)$ & $0(0.0)$ & $0(0.0)$ \\
Bile & $1(0.7)$ & $1(1.5)$ & $0(0.0)$ \\
Total & $150(100.0)$ & $43(100.0)$ & $15(100.0)$ \\
\hline
\end{tabular}

Antibiogram of ESBL -producer MDR E. coli

All ESBL-producers were highly susceptible to imipenem ( $n=135,90.0 \%)$ and meropenem ( $n=120,80.0 \%)$ followed by piperacillin/tazobactam ( $n=119,79.0 \%$ ), chloramphenicol ( $n=46,78.0 \%)$ and amikacin $(n=117$, $78.0 \%$ ) but most of the isolates were resistance to third generation cephalosporins and non- $\beta$-lactam antibiotics (Table 5). 


\begin{tabular}{|llll|}
\hline \multicolumn{4}{|l}{$\begin{array}{l}\text { Table 4. Distribution of ESBL and MBL- producer E. } \\
\text { coli strains among different } \text { wards }(\mathbf{n}=250) \text {. }\end{array}$} \\
\hline Wards & ESBL & MBL & ESBL+ MBL \\
& No. (\%) & No. (\%) & No. (\%) \\
Surgical & $80(53.3)$ & $26(60.4)$ & $9(60.0)$ \\
Medical & $13(8.7)$ & $1(2.3)$ & $1(6.7)$ \\
Neuro & $12(8.0)$ & $5(11.8)$ & $1(6.7)$ \\
ICU & $11(7.3)$ & $2(4.6)$ & $1(6.7)$ \\
Paediatric & $9(6.0)$ & $2(4.6)$ & $2(13.3)$ \\
POW & $7(5.0)$ & $3(7.0)$ & $0(0.0)$ \\
Maternity & $6(4.0)$ & $0(0.0)$ & $0(0.0)$ \\
Nephro & $5(3.3)$ & $0(0.0)$ & $0(0.0)$ \\
Orthopedics & $3(2.0)$ & $1(2.3)$ & $0(0.0)$ \\
NICU & $1(0.7)$ & $2(4.7)$ & $0(0.0)$ \\
PICU & $1(0.7)$ & $1(2.3)$ & $1(0.0)$ \\
Burn & $1(0.7)$ & $0(0.0)$ & $0(0.0)$ \\
Eye & $1(0.7)$ & $0(0.0)$ & $0(0.0)$ \\
Total & $150(100.0)$ & $43(100.0)$ & $15(100.0)$ \\
\hline
\end{tabular}

\begin{tabular}{|lll|}
\hline $\begin{array}{l}\text { Table 5. Antibiogram of ESBL producer MDR E.coli } \\
\text { (n=150). }\end{array}$ & Sensitive & Resistant \\
\hline Antibiotics & No (\%) & No (\%) \\
Imipenem & $135(90.0)$ & $15(10.0)$ \\
Meropenem & $120(80.0)$ & $30(20.0)$ \\
Piperacillin + Tazobactam & $119(79.3)$ & $31(20.7)$ \\
Chloramphenicol* * & $46(78.0)$ & $13(22.0)$ \\
Amikacin & $117(78.0)$ & $33(22.0)$ \\
Cefoperazone/ sulbactam & $84(56.0)$ & $16(44.0)$ \\
Cefoxitin & $73(48.7)$ & $77(51.3)$ \\
Gentamicin & $72(48.0)$ & $78(52.0)$ \\
Doxycycline & $43(28.6)$ & $107(71.3)$ \\
Nitrofurantoin* & $51(56.0)$ & $40(44.0)$ \\
Amoxycillin/ clavulanic & $49(32.7)$ & $101(67.3)$ \\
acid & & \\
Cefepime & $17(11.3)$ & $133(88.7)$ \\
Cotrimoxazole & $12(8.0)$ & $142(92.0)$ \\
Ciprofloxacin & $10(6.7)$ & $140(93.3)$ \\
Ofloxacin & $10(6.7)$ & $140(93.3)$ \\
Ceftazidime & $1(0.4)$ & $149(99.6)$ \\
Cefotaxime & $0(0.0)$ & $150(100.0)$ \\
Ceftriaxone & $0(0.0)$ & $150(100.0)$ \\
Amoxycillin & $0(0.0)$ & $150(100.0)$ \\
\hline
\end{tabular}

** Except urinary isolates

* Only for urinary isolates

\section{Antibiogram of MBL-producer MDR E. coli}

Antibiotic susceptibility tests of MBL producers revealed totally resistance to all penicillins and cephalosporins (including inhibitor combinations) and carbapenems. The isolates showed high percentage of resistance to most antibiotics tested: ciprofloxacin $(n=42$, $97.7 \%)$, ofloxacin ( $n=42,97.7 \%)$, gentamicin $(n=41$, $95.3 \%)$, cotrimoxazole $(n=41,95.3 \%)$, piperacillin/ tazobactam ( $n=41,95.3 \%)$, Amikacin $(n=38,88.3 \%)$, nitrofurantoin $(n=11,83.7 \%)$ and doxycycline $(n=34$, $79.1 \%)$. Polymyxins and tigecycline were the most effective antibiotics ( $n=43,100.0 \%$ ) against MBLproducer MDR E. coli followed by chloramphenicol $(n=13,52.0 \%)$.

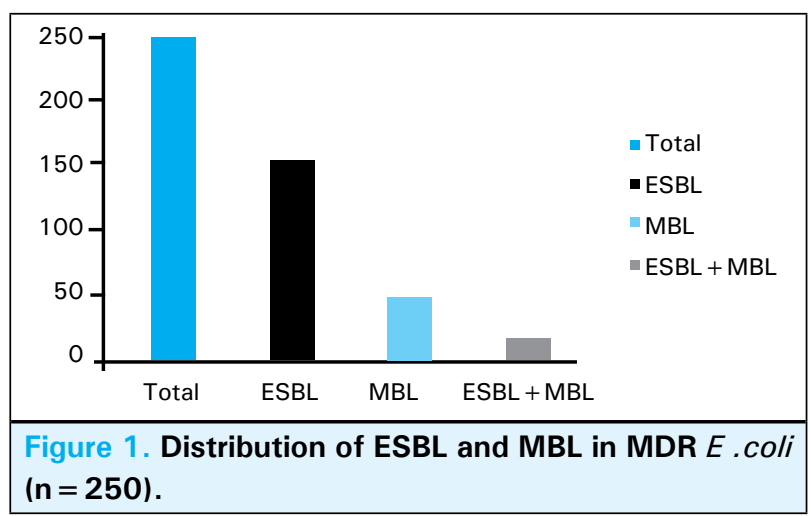

Antibiogram of ESBL and MBL co-producer MDR E. coli

All the ESBL and MBL co-producer isolates were highly sensitive $(n=15,100.0 \%)$ to polymyxins and tigecycline but completely resistant to all penicillins and cephalosporins (including inhibitor combinations), aminoglycosides, cephamycins, fluoroquinolones, folate pathway inhibitors and nitrofurantoin.

\section{DISCUSSION}

ESBL and MBL enzymes are of increasing clinical concern. ESBLs are most commonly produced by Escherichia coli and Klebsiella spp. but may also be present in other gram negative bacteria. Many MDR bacteria produce multiple $\beta$-lactamases including combinations of these different enzymes. With the increasing number of $\mathrm{MBL}$ and KPC producing bacteria and ESBL and AmpC producing bacteria associated with porin loss and efflux mechanisms, there has been an increasing resistance to carbapenems. ${ }^{4}$ Prolonged antibiotic exposure, overstay in hospitals, severe illness, unprecedented use of third generation cephalosporin, and increased use of intravenous devices or catheters are important risk factors for infection with MDR E. coli. ${ }^{12}$

The current study demonstrated that $(n=127,50.8 \%$ ) of MDR E. coli were isolated from urine samples and ( $n=141,56.4 \%$ ) from surgical wards. With regard to urinary tract infection among hospitalized patients, many researchers indicated its incidence as: $31 \%$ $47 \% .{ }^{13-15}$ In the present study, most of the patients in surgical wards have indwelling urinary catheter. The indwelling urinary catheter as an invasive device has a significant association with hospital acquired urinary tract infections for it provides either a portal of entry for microorganism or a place for colonization of microorganisms. ${ }^{16}$ 
Shrestha et al. Phenotypic Characterization of Multidrug-Resistant Escherichia Coli with Special Reference...

For all of MDR E.coli isolates, colistin, polymyxin B and tigecycline had the excellent activity followed by chloramphenicol, Piperacillin/tazobactam, amikacin and carbapenems. The resistance of $E$. coli isolates towards the third generation and fourth generation cephalosporins- cefotaxime and cefepime could be attributed to ESBL or some other relevant underlying mechanisms. Results of our study have shown that infection with ESBL producing MDR E. coli was ( $n=150,60.0 \%$ ) in our setting which was alarmingly high. Others have reported $50-70 \%$ prevalence of ESBL producing among MDR E. coli. ${ }^{17,18}$ ESBL production varies from hospital to hospital because of variation in selection of type of antibiotics. The selective pressures which are generated by the indiscriminate use of the beta-lactam antibiotics have led to the selection of a variety of mutated forms of $\beta$-lactamases such as ESBLs. ${ }^{19}$

In this study $(\mathrm{n}=43,17.0 \%)$ isolates were $\mathrm{MBL}$ producers. Various authors have reported $2.9 \%$ $25 \% \mathrm{MBL}$ producing E.coli strains from hospitalized patients. ${ }^{20-22}$ Our findings were in concordance with the studies which were done by Bora et $\mathrm{al}^{23}$ who reported $18.9 \%$ and Bandekar et al, ${ }^{24}$ who reported $15.7 \% \mathrm{MBL}$ producers. $\mathrm{MBL}$ producing organisms were isolated mainly from surgical wards $(n=26,60.4 \%)$. Indwelling medical devices are commonly used in these wards, which play a key role in the spread of infective agents. In any hospital setting, carbapenems are used as the last resort for treatment of MDR gram-negative bacterial infection. Antibiotic overuse is an important contributor for the emergence and spread of resistance; association between carbapenem consumption and resistance has been previously documented. ${ }^{25}$ However, since last 15 years, acquired resistance which is mainly mediated by MBLs to these life saving antimicrobials has been increasingly reported worldwide including Nepal not only in Pseudomonas and Acinetobacter spp, but also among members of Enterobacteriaceae. ${ }^{26,27}$

Analysis of antimicrobial susceptibility pattern of $\mathrm{ESBL}$ producing $E$. coli isolates demonstrated high susceptibility rates towards imipenem $(n=135$, $90.0 \%)$ and meropenem ( $n=120,80.0 \%)$ followed by piperacillin/tazobactam ( $n=119,79.0 \%)$, amikacin $(n=117,78.0 \%)$ and chloramphenicol $(n=46,78.0 \%)$. Similar susceptibility patterns were observed in studies conducted in Nepal and India. ${ }^{28-30} \mathrm{MBL}$ producing bacterial isolates can confer resistance to carbapenems and all beta-lactam agents except aztreonam although coexistence of other resistance mechanisms such as AmpC type beta-lactamases or ESBLs render them resistant to aztreonam. ${ }^{31}$ We observed all $\mathrm{MBL}$ producer E.coli were resistant to imipenem and meropenem. These isolates also demonstrated a high level of resistance to amoxycillin, the third and fourth generation cephalosporins, amikacin and gentamicin as well as to the beta-lactam/beta-lactamase inhibitor combination tested in the study. These findings are similar with other reports. ${ }^{23,27}$ Present study identified ESBL and MBL co-producers in $(n=15,6.2 \%)$ isolates. An increased rate of occurrence of ESBL and MBL co-producers (8.7 $\%)$ was also observed among nosocomial isolates of E.coli in a recent report from India. ${ }^{32}$ This study has demonstrated a very high level of resistance to the most of antibiotics tested in ESBL and MBL co-producer E.coli. Only polymyxins and tigecycline have potent activity against these isolates. Although the isolates were uniformly susceptible to polymyxin B and tigecycline in vitro, outcomes for infected patients treated with these agents remain unknown. ${ }^{33}$ The coexistence of different classes of $\beta$-lactamases in a single bacterial isolate may pose diagnostic and treatment challenges because the treatment options are fast running out. They are of significant concern because they create therapeutic dilemma, cause treatment failures and are increasing in occurrence worldwide. It might be undertaken that in the absence of novel agents in the near future, the spread of ESBL and MBL co-producers may lead to therapeutic dead ends.

\section{CONCLUSIONS}

Of particular concern are our results showing frequent carbapenem resistance among $E$. coli isolates, as well as the high rates of resistance to non-beta-lactam agents. This report underlined a real threat from the emergence of extreme drug-resistant and pan drug-resistant bacteria in near future. The spread of ESBL and MBL producing bacteria has been noticeably rapid worldwide including Nepal, indicating that continuous monitoring systems and effective infection control measures are absolutely required. Therapeutic options for infections due to ESBL and MBL producers have also become increasingly limited. Therefore, a better understanding of $\beta$-lactamase mediated resistance mechanisms is critical for optimizing therapy. In view of the exhaustion of available therapeutic options, investment in infection control resources and optimal antibiotic use, along with harmonized efforts from all concerned authorities is urgently required. 


\section{REFERENCES}

1. Paterson DL, Bonomo RA. Extended-Spectrum- $\beta$-Lactamases: a clinical update. Clin Microbiol Rev. 2005;18(4):657-86.

2. Livermore DM. Beta-Lactamases in laboratory and clinical resistance. Clin Microbiol Rev.1995;8(4):557-84.

3. Papp-Wallace KM, Endimiani A, Taracila MA, Bonomo RA. Carbapenems: past, present and future. Antimicrob Agents Chemother. 2011;55(11):4943-60.

4. Bush K, Fisher JF. Epidemiological expansion, structural studies, and clinical challenges of new B-lactamases from gram-negative bacteria. Annu Rev Microbiol. 2011;65:455-78.

5. Diekema DJ, Pfaller MA, Jones RN, Doern GV, Winokur PL, Gales AC, et al. Survey of bloodstream infections due to gramnegative bacilli: frequency of occurrence and antimicrobial susceptibility of isolates collected in the United States, Canada, and Latin America for the SENTRY Antimicrobial Surveillance Program,1997. Clin Infect Dis. 1999;29:595-607.

6. Yong D, Giske CG. Characterization of a new metallo- $\beta$ lactamase gene, $\mathrm{bla}_{\mathrm{NDM}-1}$, and a novel erythromycin esterase gene carried on a unique genetic structure in Klebsiella pneumoniae sequence type 14 from India. Antimicrob Agents Chemother. 2009;53:5046-54.

7. Tada T, Miyoshi-Akiyama T, Dahal RK, Sah MK, Ohara H, Kirikae T, Pokhrel BM. NDM-8 metallo- $\beta$-Lactamase in a multidrug-resistant Escherichia coli strain Isolated in Nepal. Antimicrob Agents Chemother. 2013;57(5):2394-6.

8. Isenberg HD. Clinical Microbiology Procedures Handbook. 2nd ed. Washington, D.C: ASM Press 2004.

9. Clinical Laboratory Standard Institute (CLSI): Performance standards for antimicrobial susceptibility testing; Twentysecond informational supplement document. Wayne, PA: CLSI: 2012;M100-S20.

10. Magiorakos AP, Srinivasan A, Carey RB, Carmeli Y, Falagas $\mathrm{ME}$, Giske CG, et al. Multidrug-resistant, extensively drugresistant and pandrug-resistant bacteria: an international expert proposal for interim standard definitions for acquired resistance. Clin Microbiol Infect. 2012; 18: 268-81.

11. Franclin C, Liolios L, Peleg AY. Phenotypic detection of carbapenem-susceptible metallo-beta-lactamase-producing Gram-negative bacilli in clinical laboratory.J Clin Microbiol. 2006;44:3139-44.

12. Chaudhary U, Aggrawal R. Extended-spectrum- $\beta$-lactamases (ESBL) - an emerging threat to clinical therapeutics. Indian J Med Microbiol. 2004;22(2):75-80

13. Mukherjee T, Pramod K, Gita S, Medha YR. Nosocomial infections in geriatric patients admitted in ICU. J Ind Acad Geriatrics. 2005;2:61-4.

14. Richards M, Edwards JR, Culver DH, Gaynes RP. Nosocomial infections in combined medical-surgical intensive care units in the United States. Infect Control Hosp Epidemiol. 2000;21:510-15

15. Lee YL,Thrupp LD, Friis RH, Fine M, Maleki P, Cessario TC. Nosocomial infection and antibiotic utilization in geriatric patients- A pilot prospective surveillance program in nursing facilities. Gerontology. 1992;38:223-32.
16. WHO. Prevention of hospital-acquired infection: a practical guide. 2nd ed.Geneva: $\mathrm{WHO} ; 2002$.

17. Sharma S, Bhat GK, Shenoy S. Virulence factors and drug resistance in Escherichia coli isolated from extraintestinal infection. Indian J Med Microbiol. 2007;25:369-73.

18. Goyal A, Prasad KN, Prasad A, Gupta S, Ghoshal U, Ayyagari A. Extended spectrum $\beta$-lactamases in Escherichia coli \& Klebsiella pneumoniae\& associated risk factors. Indian J Med Res. 2009;129:695-700.

19. Owens RC Jr1, Rice L. Hospital-based strategies for combating resistance. Clin Infect Dis. 2006;42 Suppl 4:S173-81.

20. Pandya NP, Prajapati SP, Mehta SJ, Kikani KM, Joshi PJ. Evaluation of various methods for detection of metallobetalactamase(MBL) production in Gram negative bacilli.Int J Biol Med Res. 2011;2(3):775-7.

21. Tsakris A,Themeli-Digalaki K, Poulou A, Vrioni G, Voulgari E, Koumaki V, et al. Comparative evaluation of combined disk tests using different boronic acid compounds for detection of Klebsiella pneumoniae carbapenemase - producing Enterobacteriaceae clinical isolates. J Clin Microbiol. 2011;49(8):2804-9.

22. Enwuru NV, Enwuru CA, Ogbonnia SO, Adepoju-Bello. Metallo-beta-lactamase production by Escherichia coli and Klebsiella species isolated from hospital and community subjects in Lagos, Nigeria. Nature and Science. 2011;9(11):1-5.

23. Bora A, Sanjana R, Jha BK, Mahaseth SN, Pokharel K. Incidence of metallo-beta-lactamase producing clinical isolates of Escherichia coli and Klebsiella pneumoniae in central Nepal. BMC Res Notes. 2014;7:557.

24. Bandekar N, Binodkumar CS, Basavarajappa KG, Prabhakar PJ, Nagaraj P. Beta- lactamases mediated resistance amongst the gram negative bacilli in burn infections. Int J Biol Res. 2011;2:766-70.

25. Iosifidis E, Antachopoulos C, Tsivitanidou M, Katraqkou A, Farmaki E, Tsiakou M, et al. Differential correlation between rates of antimicrobial drug consumption and prevalence of antimicrobial resistance in a tertiary care hospital in Greece. Infect Control Hosp Epidemiol. 2008;29(7):615-22

26. Gupta V, Datta P, Chander J. Prevalence of metallo beta- lactamase (MBL) producing Pseudomonas spp. and Acinetobacter spp. in a tertiary care hospital in India. J Infect. 2005;52:311-14

27. Mishra SK, Acharya J, Kattel HP, Koirala J, Rijal BP, Pokhrel BM. Metallo-beta-lactamase producing gram-negative bacterial isolates. J Nepal Health Res Counc. 2012;10:208-13.

28. Khanal S, Bhatta DR, Devkota U, Pokhrel BM. Betalactamase-producing multidrug-resistant bacterial pathogens from tracheal aspirates of intensive care unit patients at National Institute of Neurological and Allied Sciences, Nepal. ISRN Microbiology. 2013:5.(http://dx.doi. org/10.1155/2013/847569)

29. Kumar D, Singh AK, Ali MR, Chander Y. Antimicrobial susceptibility profile of extended-spectrum- $\beta$-lactamase (ESBL) producing Escherichia coli from various clinical samples. Infect Dis. 2014;7:1-8. 
Shrestha et al. Phenotypic Characterization of Multidrug-Resistant Escherichia Coli with Special Reference...

30. Babypadmini S, Appalaraju B. Extended-spectrum- $\beta$ lactamases in urinary isolates of E. coli and K. pneumoniaeprevalence and susceptibility pattern in a tertiary care hospital. Indian J Med Microbiol. 2004;22(3):172-4.

31. Queenan AM, Bush K. Carbapenemases: the versatile $\beta$-lactamases. Clin Microbiol Rev. 2007;20(3):440-58.
32. Oberoi L, Singh N, Sharma P, Aggarwal A. ESBL, MBL, AmpC $\beta$-Lactamases Producing Superbugs - Havoc in the intensive care units of Punjab, India. J Clin Diag Res. 2013;7(1):70-3.

33. Urban C, Bradford PA, Tuckman M, Segal-Maurer S, Wegbeh W, Grenner L, et al. Carbapenem-resistant Escherichia coli harboring Klebsiella pneumoniae carbapenemase-betaLactamases associated with long-term care facilities. Clin Infect Dis. 2008;46(1):127-30. 